\title{
Efficacy and safety evaluation of laparoscopic D3 lymphadenectomy combined with pelvic autonomic nerve preservation for rectal can-
}

\section{cer}

\author{
Ting Li*, Xiang-ling Meng, Zhen Zhang \\ General surgical department, the First Affiliated Hospital of Anhui Medical University, Hefei 230022, China
}

\begin{abstract}
Background: Laparoscopic D3 lymphadenectomy is widely used in rectal cancer treatment. This study aimed to investigate the efficacy and safety of the laparoscopic D3 lymphadenectomy combined with pelvic autonomic nerve preservation in treating rectal cancer.

Methods: We enrolled 211 patients with rectal cancer who admitted to our hospital from 2012 to 2016.

Randomly, 131 cases underwent laparoscopic D3 lymphadenectomy (laparoscope group) and 80 cases underwent open surgery (open group). The patients' clinical data and statistical results were retroactively comparatively analyzed.

Results: All the operations were completed successfully in 211 patients. No significant differences were found between the two groups in the number of removed lymph nodes and postoperative complications. The carminative time and time to get out of bed were shorter in the laparoscope group than that in the open group. The hospital stay was also shorter in the laparoscope group than in the open group, while the surgical duration was shorter in the open group. No significant differences were observed between the two groups in recurrence rates, mortality rates, or postoperative urinary and sexual dysfunction.

Conclusion: The laparoscopic D3 lymphadenectomy combined with pelvic autonomic nerve preservation is feasible and safe for rectal cancer treatment. The advantages include being minimally invasive and a quicker postoperative recovery, which improves the patient's quality of life.

Keywords: rectal cancer; laparoscope; D3 lymphadenectomy; pelvic autonomic nerve preservation
\end{abstract}

\section{INTRODUCTION}

Rectal cancer is a very common gastrointestinal cancer with a high malignancy rate and unknown etiology ${ }^{[1]}$. Even worse, rectal cancer is a leading cause of cancer-related deaths worldwide and may occur in younger people $^{[2]}$. Due to the extraordinary location of rectal cancer, it is impossible to remove the tumor thoroughly, which can bring many challenges for treatment and can lead to a high recurrence rate ${ }^{[3]}$. With the many advances made in the management of rectal cancer, higher requirements have been proposed for surgical treatment, including minimally invasive procedures and a higher quality of life after the operation. Thus, this emphasizes the importance of the D3 lymphadenectomy procedures ${ }^{[4]}$. Lymph node metastasis is the primary spreading pathway of rectal cancer; hence, a lymph node dissection is an effec-

*Corresponding author: Ting Li

Mailing address: General surgical department, the First Affiliated Hospital of Anhui Medical University, Hefei 230022, China Address: Jixi Road No.218, Hefei, Anhui, 230022, China E-mail: tingli_022@163.com tive way to increase the survival rate. A D3 lymph node dissection can be performed with good technical efficiency, a quick functional recovery, and mild disability for patients with distal rectal cancer ${ }^{[5]}$.

With the enhanced development of laparoscopic approaches, minimally invasive surgeries using these techniques have become more common in the general surgery for gastrointestinal tumors. The laparoscopic approach, while still a debatable topic for rectal cancer, is currently accepted as an alternative to open surgery for colon cancer ${ }^{[6]}$. Furthermore, it has long been shown to have short-term benefits and be oncologically safe ${ }^{[7]}$. It has been challenging applying laparoscopic approaches to perform a D3 lymphadenectomy, which requires advanced laparoscopic skills as well as comprehensive anatomical knowledge and sufficient experience with minimally invasive operations ${ }^{[8]}$. This surgery is extensive in scope and involves several important organs, and a deep insight into the superior mesenteric vein anatomy is necessary to achieve a complete removal ${ }^{[9]}$.The primary task of surgical intervention for rectal cancer is to realize an oncologic cure as well as maintain sexual 
Table 1 Clinic data of patients in the two groups

\begin{tabular}{|c|c|c|c|c|c|c|c|c|c|}
\hline \multirow{2}{*}{ Groups } & \multirow{2}{*}{ Cases } & \multirow{2}{*}{ Ages (mean \pm SD) } & \multicolumn{2}{|c|}{ Genders } & \multicolumn{2}{|c|}{ Types of surgery } & \multicolumn{3}{|c|}{ TNM staging } \\
\hline & & & Male & Female & Dixon & Miles & I stage & II stage & III stage \\
\hline Laparoscope group & 131 & $33-65(53.05 \pm 9.25)$ & 78 & 53 & 91 & 40 & 16 & 87 & 28 \\
\hline Open group & 80 & $26-65(53.56 \pm 8.32)$ & 45 & 35 & 59 & 21 & 12 & 50 & 18 \\
\hline$t / x 2$ & & 0.5 & 0.22 & & 0.44 & & 0.44 & & \\
\hline$P$ value & & $>0.05$ & $>0.05$ & & $>0.05$ & & $>0.05$ & & \\
\hline
\end{tabular}

and urinary functions, for which pelvic autonomic nerve preservation is indispensable in minimizing nerve damage and improving quality of life $^{[10]}$. However, the rectum is located concavely along the curved sacrum and both the ischial tuberosity and iliac wing limit the pelvic cavity boundary, which increases the difficulty of pelvic autonomic nerve preservation ${ }^{[11]}$. For colorectal surgeons, a lack of anatomical knowledge and poor visualization of the pelvic autonomic nerves are the main causes for intraoperative nerve injury, which is the primary reason for sexual and urinary dysfunction. Fortunately, combined use of the laparoscopic D3 lymphadenectomy may be a benefit for surgeons in completing the pelvic autonomic nerve preservation. Thus, with a retrospective study, and aiming to draw lessons from the previous experience, we investigated the efficacy and safety of laparoscopic D3 lymphadenectomy combined with pelvic autonomic nerve preservation in treating rectal cancer.

\section{METHODS}

\section{Clinical data}

A total of 211 patients with rectal cancer admitted to our hospital from 2012 to 2016 and were enrolled in this study. The patients were randomly divided into a laparoscope group $(n=131)$, who underwent laparoscopic D3 lymphadenectomy, and an open group ( $\mathrm{n}=$ 80), who underwent open surgery. The inclusion criteria were patients with no previous history of sexual or urinary dysfunction and no history of chemotherapy or radiotherapy. Patients with distant metastasis and those who could not tolerate surgery were excluded. All patients agreed and signed the documented informed consent form for this study before treatment. This research was approved by the local ethics committee and was performed in accordance with the Declaration of Helsinki. Basic data, such as age, gender, and surgery type, as well as tumor, node, and metastasis (TNM) staging are displayed in Table 1.

\section{Surgical procedure}

After an abdominal exploration, the posterior peritoneum was incised using an ultrasound knife and the nearby lymph and fat tissue were removed, and then the inferior mesenteric vessels at the root was excised.
The sigmoid mesocolon was separated, with the genital blood vessels and ureter carefully protected. A rectal low anterior resection (Dixon) was performed ${ }^{[12]}$. Briefly, the mesorectum was dissociated according to the principle of a total mesorectal excision, avoiding damage in the inferior hypogastric plexus. The collateral ligament is where the gonad and bladder nerves derived from the pelvic autonomic nerve, and the gonad and bladder nerves were cared for when dissociated the collateral ligament. The levator ani muscle was exposed and the intestinal canal was excised using a linear cut stapler; the cut of the proximal colon was treated with a purse string suture. The anastomat was put through the anus, and the anastomosis between the rectum and colon was completed with a laparoscopy. The procedures for rectal separation in Miles surgery were the same as those described above, and the sample was taken out through the incision in the perineum. The middle incision at the left side was used in the open group, sharing the same surgical procedures and dissection ranges with the laparoscopic group.

\section{Observational index}

The surgery duration, the postoperative time to get out of bed, the amount of removed lymph node, the postoperative carminative time, postoperative complications, and postoperative urinary and sexual functions were observed and compared between the two groups. The sexual function of males was distinguished into three grades: 0 grade: with erection of the penis, sexual intercourse, and ejaculation; I grade: with erection of the penis, sexual intercourse, but without ejaculation; and II grade: impotence. Urinary function was also divided into three grades: 0 grade: self-maturate after pulling out the catheter, and with $<50 \mathrm{ml}$ of residual urine volume; I grade: with $50 \sim 100 \mathrm{ml}$ of residual urine volume after pulling out the catheter; and II grade: with $50 \sim 100 \mathrm{ml}$ of residual urine volume after pulling out the catheter, or the catheter was absolutely necessary. All cases were followed up for 1 52 months to monitor any tumor recurrence.

\section{Statistical analysis}

All the statistical data were analyzed with SPSS 21.0 software. The enumeration data were analyzed with 2 
Table 2 Patients' conditions during the surgery in the two groups

\begin{tabular}{lll}
\hline Groups & $\begin{array}{l}\text { Duration of } \\
\text { operation }\end{array}$ & $\begin{array}{l}\text { Amount of removed } \\
\text { lymph nodes }\end{array}$ \\
\hline Laparoscope group & $3.33 \pm 0.72$ & $14.94 \pm 3.41$ \\
Open group & $2.83 \pm 0.62$ & $15.35 \pm 3.13$ \\
$\mathrm{t} / \mathrm{x} 2$ & 5.14 & 0.88 \\
P value & $<0.01$ & $>0.05$ \\
\hline
\end{tabular}

tests, and the measurement data were evaluated with student's t-test. A P value of $<0.05$ was considered significant.

\section{RESULTS}

\section{Patients' conditions during the surgery}

All the operations were completed successfully in 211 patients, and without any operative death. While there was no significant difference between the two groups in the number of removed 1ymph nodes, the surgical duration was shorter in the open group (Table 2).

\section{Patients' postoperative situations}

The carminative time and the time to get out of bed were shorter in the laparoscope group than in the open group. Also, the hospital stay was shorter in the laparoscope group than in the open group. However, no significant difference was found between the two groups in the incidence of postoperative complications (Table 3).

\section{Follow-up and therapeutic evaluation}

All cases were followed up for 1 52 months to monitor the tumor recurrence. No significant differences were observed between the two groups in recurrence rate, mortality rate, and postoperative urinary and sexual dysfunction (Table 4).

\section{DISCUSSION}

OA curative resection with a lymphadenectomy is widely believed to be the standard therapeutic method for resectable colorectal cancer, but the best method of lymph node dissection has not been normalized yet ${ }^{[13]}$. Several retrospective studies have demonstrated that a D3 lymphadenectomy contributes to reducing both the local recurrence and distant metastasis and ameliorates the survival of patients with rectal cancer ${ }^{[5]}$. Furthermore, the laparoscopic approach results in a surgery that is, to a large extent, minimally invasive ${ }^{[14]}$. With the amplification effect of the laparoscope, the D3 lymphadenectomy achieves a thorough dissection of the involved lymph nodes and prevents distant metastasis and recurrence of the tumor. The preservation of the pelvic autonomic nerves protects the patients from urinary and sexual dysfunction after the surgery, thus, enabling them to conduct a normal life and ensure their quality of life. We showed that laparoscopic D3 lymphadenectomy combined with pelvic autonomic nerve preservation is feasible and safe for rectal cancer treatment. An analysis of the clinical data suggested that patients treated with the laparoscope got out of the bed earlier, which is a benefic in the recovery of intestinal function and prevention of lung complications. Moreover, the laparoscope procedure shortened the hospital stay of patients, thus exhibiting its merit in being minimally invasive. No significant differences were observed between the two groups in postoperative urinary and sexual dysfunction, which indicates that the pelvic autonomic nerve preservation combined with the laparoscope effectively protected the urinary and sexual functions and improved the quality of life of the patients. The results of follow-up examinations showed no significant difference between the two groups in recurrence rate, implying no clear difference between the laparoscope and open surgery in curing rectal cancer.

With the rapid development of laparoscopic approaches, the laparoscopic D3 lymphadenectomy combined with pelvic autonomic nerve preservation exhibited unique advantages in many aspects, and some matters should be paid more attention ${ }^{[15]}$. Firstly, the amplification effect of the laparoscope makes it easier to distinguish the smaller lymph nodes and increase the efficiency and thoroughness of lymph node dissection ${ }^{[16]}$. Secondly, the dissection and cutting of the inferior mesenteric vessels interdicts the pathway of tumor hematogenous metastasis. Moreover, this was handled without contact with the tumor, minimizing the press to the tumor, which agrees with the principle of a tumor-free technique ${ }^{[17]}$. Thirdly, the amplification effect of the laparoscope guarantees the accuracy of the operative approach and anatomical level, and the trend and smaller branch of the pelvic autonomic nerve can be clearly identified, avoiding the damage of the pelvic autonomic nerve during the oper-

Table 3 Clinic data of patients in the two groups

\begin{tabular}{|c|c|c|c|c|c|c|c|c|}
\hline \multirow[b]{2}{*}{ Groups } & \multirow{2}{*}{$\begin{array}{l}\text { Postoperative } \\
\text { carminative } \\
\text { time }\end{array}$} & \multirow{2}{*}{$\begin{array}{l}\text { Time of get } \\
\text { out of bed } \\
\text { after surgery }\end{array}$} & \multirow{2}{*}{$\begin{array}{l}\text { Postoperative } \\
\text { complications }\end{array}$} & \multicolumn{4}{|c|}{ Types of postoperative complications } & \multirow{2}{*}{$\begin{array}{l}\text { Hospital } \\
\text { stay }(d)\end{array}$} \\
\hline & & & & $\begin{array}{l}\text { Incision } \\
\text { infection }\end{array}$ & $\begin{array}{l}\text { Pulmonary } \\
\text { infection }\end{array}$ & $\begin{array}{l}\text { Anastomotic } \\
\text { fistula }\end{array}$ & $\begin{array}{l}\text { Anastomotic } \\
\text { bleeding }\end{array}$ & \\
\hline Laparoscope group & $3.48 \pm 0.82(d)$ & $1-4(d)$ & $16(12.2 \%)$ & $9(56.3 \%)$ & $2(12.5 \%)$ & $1(6.3 \%)$ & $4(25 \%)$ & $16.62 \pm 4.55$ \\
\hline Open group & $2.83 \pm 0.62(d)$ & $3-5(d)$ & $11(13.8 \%)$ & $6(54.5 \%)$ & $2(18.2 \%)$ & $2(18.2 \%)$ & $1(9.1 \%$ & $18.31 \pm 6.07$ \\
\hline$t / x 2$ & 4.86 & 8.49 & 0.1 & 1.87 & & & & 2.31 \\
\hline$P$ value & $<0.01$ & $<0.01$ & $>0.05$ & $>0.05$ & & & & $<0.05$ \\
\hline
\end{tabular}


Table 4 Clinic data of patients in the two groups

\begin{tabular}{llllllllll}
\hline \multirow{2}{*}{ Groups } & $\begin{array}{l}\text { Recurrence } \\
\text { rate (\%) }\end{array}$ & $\begin{array}{l}\text { Mortality } \\
\text { rate (\%) }\end{array}$ & \multicolumn{2}{l}{ Urinary dysfunction } & \multicolumn{3}{l}{ Sexual dysfunction } \\
\cline { 5 - 9 } & & 0 degrade & I degrade & II degrade & Males & $\begin{array}{l}0 \text { degrade } \\
(\%)\end{array}$ & $\begin{array}{l}\text { I degrade } \\
(\%)\end{array}$ & $\begin{array}{l}\text { II degrade } \\
(\%)\end{array}$ \\
\hline Laparoscope group & $15(11.45)$ & $6(4.58)$ & 108 & 23 & 0 & 78 & $63(80.7)$ & $13(16.7)$ & $2(2.6)$ \\
Open group & $9(11.25)$ & $3(3.75)$ & 67 & 13 & 0 & 45 & $36(80.0)$ & $7(15.6)$ & $2(4.4)$ \\
t/x2 & 0.01 & 0.08 & 0.06 & & & & 0.32 & \\
P value & $>0.05$ & $>0.05$ & $>0.05$ & & & & $>0.05$ & \\
\hline
\end{tabular}

ation ${ }^{[18]}$. Fourthly, the hypogastric nerves are most likely to be damaged at the sacral promontory, from which two branches stretch to peripheral nerves with many reticular structures, and they should be given closer consideration during surgery with the right stratified anato$\mathrm{my}^{[19]}$. Finally, the collateral ligament is where the gonad and bladder nerves derived from the pelvic autonomic nerve, and the gonad and bladder nerves should be protected when dissociated the collateral ligament ${ }^{[20]}$.

The laparoscopic D3 lymphadenectomy combined with pelvic autonomic nerve preservation not only removes lymph nodes that may cause skip metastasis ${ }^{[21]}$, but also preserves the pelvic autonomic nerve ${ }^{[11,22]}$, enhancing survival rates and improving the postoperative quality of life. It also facilitates a rapid recovery after surgery, confirming its safety and efficacy in clinical applications in treating rectal cancer. As a promising minimally invasive surgery, the use of the laparoscopic D3 lymphadenectomy combined with pelvic autonomic nerve preservation deserves to be popularized in the future, with skilled operation techniques needed for colorectal surgeons.

Our retrospective study has some limitations, including the inherent weakness of being observational and non-experimental. In addition, very few studies have been published concerning a large number of patients who have undergone D3 lymphadenectomy for rectal cancer until now. Therefore, further studies on a greater number of cases are necessary to validate the value of the D3 lymphadenectomy combined with pelvic autonomic nerve preservation in treating rectal cancer.

\section{CONFLICT OF INTEREST}

The authors declare no conflict of interest.

\section{REFERENCES}

1. Yu, S. K., Bhangu, A., Tait, D. M., Tekkis, P., Wotherspoon, A., and Brown, G. (2014) Chemoradiotherapy response in recurrent rectal cancer. Cancer Med 3, 111-117

2. Gaertner, W. B., Kwaan, M. R., Madoff, R. D., and Melton, G. B. (2015) Rectal cancer: An evidence-based update for primary care providers. World J Gastroenterol 21, 76597671

3. Troja, A., Petersen, S., Antolovic, D., and Raab, H. R. (2014) [Limitations of surgery for rectal cancer]. Chirurg 85, 192-
197

4. Ubukata, M., Itabashi, M., and Kameoka, S. (2014) Japanese D3 lymph node dissection in low rectal cancer with inferior mesenteric lymph node metastases. World J Surg $38,2724-2729$

5. Liang, J. T., and Lai, H. S. (2014) Surgical technique of robotic D3 lymph node dissection around the inferior mesenteric artery with preservation of the left colic artery and autonomic nerves for the treatment of distal rectal cancer. Surg Endosc 28, 1727-1733

6. Zhao, L. Y., Liu, H., Wang, Y. N., Deng, H. J., Xue, Q., and Li, G. X. (2014) Techniques and feasibility of laparoscopic extended righthemicolectomy with D3 lymphadenectomy. World J Gastroenterol 20, 10531-10536

7. Shussman, N., and Wexner, S. D. (2014) Current status of laparoscopy for the treatment of rectal cancer. World J Gastroenterol 20, 15125-15134

8. Liang, J. T., Huang, K. C., Lai, H. S., Lee, P. H., and Sun, C. T. (2007) Oncologic results of laparoscopic D3 lymphadenectomy for male sigmoid and upper rectal cancer with clinically positive lymph nodes. Ann Surg Oncol 14, 1980-1990

9. Li, Y. (2015) Laparoscopic extended right hemicolectomy with D3 lymphadenectomy. Ann Transl Med 3, 124

10. Kim, N. K., Kim, Y. W., and Cho, M. S. (2015) Total mesorectal excision for rectal cancer with emphasis on pelvic autonomic nerve preservation: Expert technical tips for robotic surgery. Surg Oncol 24, 172-180

11. Grama, F. A., Burcos, T., Bordea, A., and Cristian, D. (2014) Localisation and preservation of the autonomic nerves in rectal cancer surgery - technical details. Chirurgia (Bucur) 109, 375-382

12. Lin, C., Zhang, Z., Wang, Y., Huang, S., Wang, L., and Wang, B. (2014) [Continuous negative pressure-flush through extraperitoneal dual tube in the treatment and prevention for rectal cancer patients with anastomotic leakage after low anterior resection]. Zhonghua Wei Chang Wai Ke Za Zhi 17, 469-472

13. Chen, H., Wang, Y., Liu, H., Hu, Y., Zhao, L., Li, G., and Chi, P. (2015) Factors influencing apical node metastasis in colorectal cancer patients treated with laparoscopic radical resection with $\mathrm{D} 3$ lymphadenectomy: results from two centers in China. Surg Today 45, 569-575

14. Han, F. H., Hua, L. X., Zhao, Z., Wu, J. H., and Zhan, W. H. (2013) Transanal natural orifice specimen extraction for 
laparoscopic anterior resection in rectal cancer. World J Gastroenterol 19, 7751-7757

15. Liu, L. Y., Zhang, C., Yu, P.W., Li, Y., Liu, T., and Xu, J. H. (2009) [Male sexual function after $\mathrm{D}(3)$ lymphadenectomy combined with pelvic autonomic nerve preservation by laparoscopic and open surgery for rectal cancer]. Zhonghua Wei Chang Wai Ke Za Zhi 12, 236-238

16. Numata, M., Hasuo, K., Hara, K., Maezawa, Y., Kazama, K., Inari, H., Takata, K., Jin, Y., Yukawa, N., Oshima, T., Rino, Y., Taguri, M., and Masuda, M. (2015) A propensity scorematching analysis comparing the oncological outcomes of laparoscopic and open surgery in patients with Stage I/II colon and upper rectal cancers. Surg Today 45, 700-707

17. Takahashi, H., Haraguchi, N., Nishimura, J., Hata, T., Matsuda, C., Yamamoto, H., Mizushima, T., Doki, Y., and Mori, M. (2017) Laparoscopic lymph node dissection around the inferior mesenteric artery for left-sided colon and rectal cancer. Surg Today

18. Sawada, T., Matoba, S., and Hayakawa, T. (2002) [Present status and problems of laparoscopic surgery for colorectal cancer, especially indications and limitations]. Nihon

\section{Geka Gakkai Zasshi 103, 742-745}

19. Maeda, Y., Shinohara, T., Nagatsu, A., Futakawa, N., and Hamada, T. (2014) Laparoscopic resection aided by preoperative 3-D CT angiography for rectosigmoid colon cancer associated with a horseshoe kidney: A case report. Asian J Endosc Surg 7, 317-319

20. Lin, M., Chen, W., Huang, L., Ni, J., Ding, W., and Yin, L. (2011) The anatomic basis of total mesorectal excision. Am J Surg 201, 537-543

21. Kobayashi, M., Okamoto, K., Namikawa, T., Okabayashi, T., and Araki, K. (2006) Laparoscopic lymph node dissection around the inferior mesenteric artery for cancer in the lower sigmoid colon and rectum: is D3 lymph node dissection with preservation of the left colic artery feasible? Surg Endosc 20, 563-569

22. Fang, J. F., Wei, B., Zheng, Z. H., Chen, T. F., Huang, Y., Huang, J. L., Lei, P. R., and Wei, H. B. (2015) Effect of intra-operative autonomic nerve stimulation on pelvic nerve preservation during radical laparoscopic proctectomy. Colorectal Dis 17, 0268-276 